\title{
Injection modelocked Ti-sapphire laser with discretely variable pulse repetition rates to $1.56 \mathrm{GHz}$
}

\author{
Curt Hovater, Matt Poelker* \\ Thomas Jefferson National Accelerator Facility, Jefferson Lab, Mailstop 16, 12000 Jefferson Ave., Newport News, VA 23606, USA
}

Received 15 September 1997

\begin{abstract}
A gain-switched diode laser was used to injection modelock a Ti-sapphire laser. The pulse repetition rate of the modelocked Ti-sapphire laser was varied by setting the diode laser pulse repetition rate equal to different multiples of the Ti-sapphire laser cavity fundamental frequency. Pulse repetition rates from $223 \mathrm{MHz}$ (fundamental) to $1.56 \mathrm{GHz}$ (seventh harmonic) were observed. No intracavity modelocking device was required and the Ti-sapphire laser cavity length was not changed. Maximum average output power at $854 \mathrm{~nm}$ was $700 \mathrm{~mW}$ for all pulse repetition rates when pumped with $6 \mathrm{~W}$ from an argon-ion laser. Pulsewidths ranged from 21 to $39 \mathrm{ps}$ (FWHM). Phase noise measurements indicate that timing jitter was $2.5 \mathrm{ps}$ at a pulse repetition rate of $223 \mathrm{MHz}$. Pulse-to-pulse amplitude fluctuations however, were significant and suggest that active cavity length stabilization may be required for reliable photoinjector applications. (C) 1998 Elsevier Science B.V. All rights reserved.
\end{abstract}

At many electron accelerators throughout the world, there is great interest in developing high duty factor, high average power $(>1 \mathrm{~W})$ laser systems with picosecond pulsewidths and repetition rates synchronized to the accelerating cavity $\mathrm{RF}$ frequency [1]. Such a laser system can be used to extract electrons from a high-voltage semiconductor-photocathode electron gun during the portion of the RF cycle when electrons are accelerated into the machine. In this way, all of the photoemitted electrons are accelerated and delivered to the ultimate user of the beam; none are wasted as is the case when a DC laser light source is used to create

\footnotetext{
*Corresponding author. Tel.: + 17572697357 ; fax: + 1757269 5279; e-mail: poelker@jlab.org.
}

a DC electron beam that must be chopped and bunched prior to injection into the accelerator. This efficient use of the extracted electrons helps to prolong the effective operating lifetime of the photocathode. Laser systems for this application must emit pulses with gigahertz repetition rates. For example, at Thomas Jefferson National Accelerator Facility (Jefferson Lab), a diode laser system with a pulse repetition rate of $1.497 \mathrm{GHz}$ provides electron beam to three nuclear physics experiment halls [2,12]. Electron accelerators such as the Mainz Microtron at Mainz, Germany and the MIT Bates Linear Accelerator require pulse repetition rates of 2.445 and $2.856 \mathrm{GHz}$, respectively. The next generation of electron accelerators may require pulsed laser systems with comparable, if not higher, repetition rates [3]. Other accelerator applications 
such as Compton polarimetry [4] and gamma-ray photon sources [5] may also benefit from the availability of high average power, high repetition rate laser systems.

Diode laser systems meet many of the pulse requirements described above but provide an average output power less than $500 \mathrm{~mW}[6,13]$. Commercial modelocked Ti-sapphire lasers are high power, tunable light sources, however, their pulse repetition rates are typically less than $100 \mathrm{MHz}$. These lasers are designed to emit high peak power, femtosecond pulses and this necessitates that the cavity length be long enough to accommodate optics (for example, prism pairs) used to compensate for group velocity dispersion within the Ti-sapphire crystal. In a recent publication, Hoffmann et al. describe a small, Kerr-lens modelocked Ti-sapphire laser with high average power and a pulse repetition rate of $1.039 \mathrm{GHz}$ [7]. Kerr-lens modelocked lasers, however, sometimes cease pulsing and must be "restarted", a condition unacceptable for reliable photoinjection. Basu et al. used a gain-switched diode laser to injection seed a Ti-sapphire laser pumped with a $Q$-switched, frequency-doubled $\mathrm{Nd}$ :YAG laser [8]. They obtained $19.4 \mathrm{ps}$ (FWHM) pulses at a rate of $200 \mathrm{MHz}$ within the $Q$-switched macropulse. In a similar manner, we report using a gain-switched diode laser to modelock a slightly modified, commercial standing-wave Ti-sapphire laser. In contrast to work reported in Ref. [8], the pulse repetition rate of the modelocked Ti-sapphire laser was varied by setting the diode seed laser repetition rate equal to different multiples of the Ti-sapphire laser cavity fundamental frequency. Pulse repetition rates from $223 \mathrm{MHz}$ to $1.56 \mathrm{GHz}$ were observed with $700 \mathrm{~mW}$ average output power for all repetition rates. In this manner, gigahertz repetition rates were obtained with a manageable cavity length (i.e., $67 \mathrm{~cm}$ rather than $<15 \mathrm{~cm})$. No intracavity modelocking elements were necessary. The gain-switched diode laser served as a simple, stable master oscillator; it is a trivial matter to obtain gain-switched pulse repetition rates to $4 \mathrm{GHz}$ [9] suggesting that operation at even higher repetition rates may be achieved with this method. The Ti-sapphire laser described here was not actively stabilized yet phase noise measurements indicate timing jitter was rela- tively low; $2.5 \mathrm{ps}$ at a $223 \mathrm{MHz}$ pulse repetition rate. It is possible active cavity length stabilization may reduce timing jitter and also decrease observed amplitude fluctuations.

Experiments were performed with a modified commercial Ti-sapphire laser (Spectra Physics Model 3900; see Fig. 1). The flat high-reflector(HR) mirror supplied with the laser was replaced with a $2 \%$ transmissive "input" coupler, which served as an input port for the seed laser beam. Both the output and input coupler mirrors were wedged at $2^{\circ}$ to avoid etalon effects that might cause undesirable optical feedback. The Ti-sapphire crystal (20 $\mathrm{mm} \times 5 \mathrm{~mm}$ dia.) was mounted on a watercooled copper heat sink. The pump laser was focused into the crystal with a $30 \mathrm{~cm}$ radius of curvature mirror; approximately $80 \%$ of the pump laser light was absorbed. The birefringent tuning filter was removed; the free running Ti-sapphire laser operated at $\approx 852 \mathrm{~nm}$. The Ti-sapphire laser emitted $700 \mathrm{~mW}$ through the output coupler mirror (5\% transmissive) and $300 \mathrm{~mW}$ through the input coupler when pumped with $6 \mathrm{~W}$ of green light from a multiline argon-ion laser. The pump laser

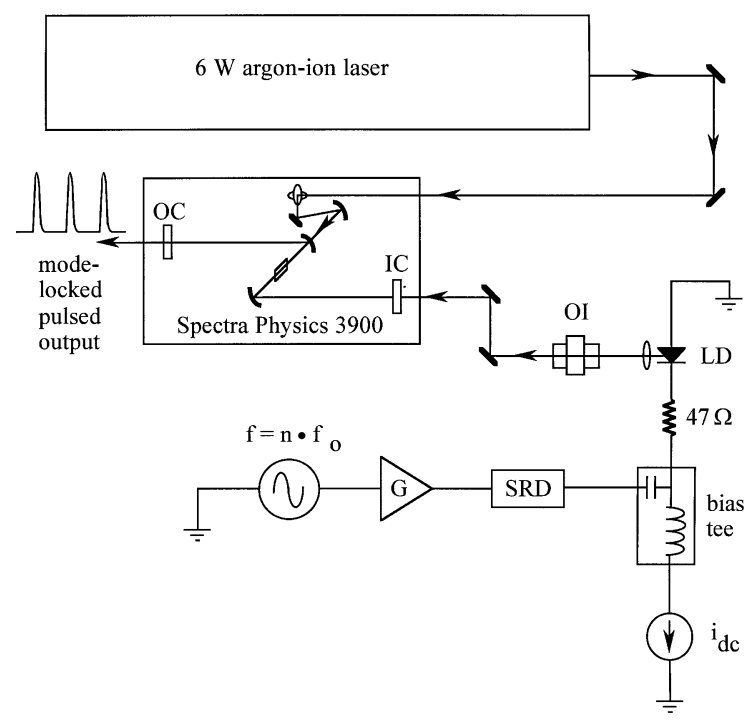

Fig. 1. Schematic representation of the injection modelocked Ti-sapphire laser. $f_{\mathrm{o}}=223 \mathrm{MHz}$, the Ti-sapphire laser cavity fundamental frequency; OI, optical isolator; OC, output coupler; IC, input coupler; SRD, step recovery diode; $L D$, laser diode. 
threshold power was approximately $2 \mathrm{~W}$. The cavity length was $67 \mathrm{~cm}$ as determined by observing the beat signal between different longitudinal modes using a fast photodiode (Optoelectronic Model PD-15) and an RF spectrum analyzer.

The seed laser (SDL 5410-G1) was gain-switched the usual way [9]. The laser was biased near threshold and a RF signal ( $\approx 1 \mathrm{~W}$ ) of the appropriate frequency was added using a bias-tee network. Comparable gain-switched diode laser performance was obtained with or without a step recovery diode (i.e., comb generator) following the RF amplifier (see Fig. 1). A $47 \Omega$ resistor was used for impedance matching, although this element also could be removed without degradation of gainswitched diode laser performance. It was important to operate the diode laser within a narrow range of DC bias current. Beyond this range, pulsewidths broadened and/or secondary pulses were observed with the fast photodiode and sampling oscilloscope (Tektronix Model 11801B with SD-32 sampling head). The average output power from the gainswitched diode laser was approximately $5 \mathrm{~mW}$ although there was some variation associated with pulse repetition rate. The wavelength of the diode laser output was $\approx 859 \mathrm{~nm}$, roughly $7 \mathrm{~nm}$ different from the free-running Ti-sapphire laser wavelength. The gain-switched diode laser output passed through an optical isolator (approximately $40 \mathrm{dBm}$ isolation) and then was directed into the Ti-sapphire laser cavity through the $2 \%$ transmissive input coupler. Care was taken to orient the polarization of the diode laser light parallel to the $c$-axis of the Ti-sapphire crystal.

The Ti-sapphire laser output went from DC to pulsed modelocked when the seed laser was aligned to ensure proper spatial modematching between the two lasers and the pulse repetition rate of the seed laser was set to within $10 \mathrm{kHz}$ of the Tisapphire laser cavity fundamental frequency (or harmonic). It is believed that modelocking occurs as a result of gain modulation caused by the presence of the seed laser beam within the Ti-sapphire laser crystal. The seed laser wavelength was within $7 \mathrm{~nm}$ of the peak of the Ti-sapphire laser gain profile; the pulsed seed laser extracts gain from the Ti-sapphire laser crystal which effectively serves to provide period loss in a manner similar to

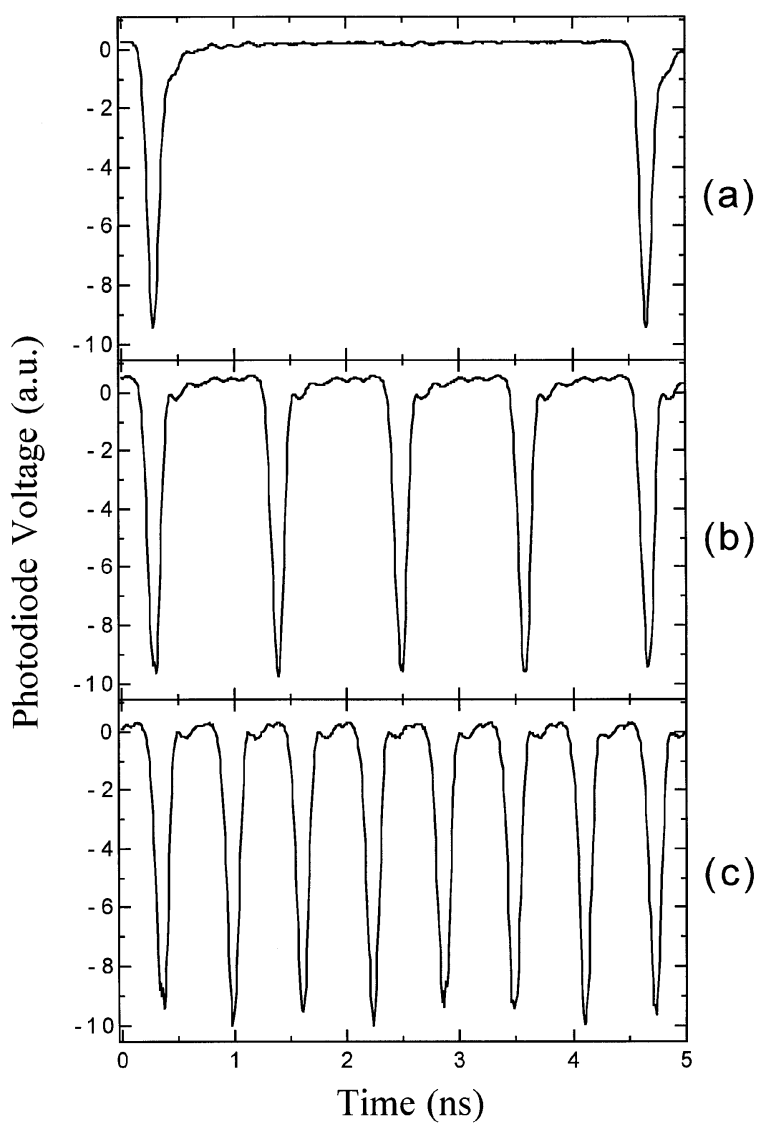

Fig. 2. Photodiode signals for three different pulse repetition rates; (a) $223 \mathrm{MHz}$, (b) $892 \mathrm{MHz}$, and (c) $1.56 \mathrm{GHz}$.

an acoustooptic modulator in a conventional modelocked laser. Pulsed operation was clearly evident from the RF spectrum of the output detected with the fast photodiode. The photodiode output was also viewed with a fast digitizing oscilloscope (Fig. 2). It was a simple matter to obtain pulse repetition rates equal to harmonics of the Ti-sapphire laser cavity fundamental frequency; only the pulse repetition rate of the seed laser was changed. We observed stable pulse repetition rates up to $1.56 \mathrm{GHz}$, the seventh harmonic of the Ti-sapphire laser cavity fundamental frequency. The modelocked average output power remained the same compared with DC operation; maximum average power through the output coupler was $700 \mathrm{~mW}$ with $6 \mathrm{~W}$ pump power. The wavelength of 
the modelocked Ti-sapphire laser output was $854 \mathrm{~nm} ; 2 \mathrm{~nm}$ different from the free-running wavelength and $5 \mathrm{~nm}$ different from the seed laser wavelength [10]. No attempt was made to determine the minimum seed laser power required to induce modelocked operation but it was apparent that $5 \mathrm{~mW}$ was considerably more than necessary. Unfortunately, the laser in its present state suffers from severe pulse-to-pulse amplitude noise with a bandwidth of approximately $40 \mathrm{kHz}$. There were brief periods ( $\approx$ minutes) of amplitude-stable pulsed operation. Typically, however, slight adjustments to the seed laser alignment were necessary and/or the seed laser pulse repetition rate needed to be adjusted by several $\mathrm{kHz}$ to reestablish amplitude-stable pulsed output.

Autocorrelator measurements were performed to determine gain-switched diode seed laser and modelocked Ti-sapphire laser pulsewidths. The autocorrelator (Femtochrome Model FR-103XL) relies on non-colinear, background-free second harmonic generation within a $\mathrm{LiIO}_{3}$ crystal. Autocorrelator traces for both the seed laser and the modelocked Ti-sapphire laser at a pulse repetition rate of $223 \mathrm{MHz}$ are shown in Fig. 3. The pulsewidths are close to Gaussian. The seed laser trace exhibits sharp spikes typical of autocorrelation measurements of gain-switched diode lasers. The spikes are separated by the round-trip time of the diode laser cavity and are indicative of the random phase relationship that exists between the different longitudinal modes excited during the gain-switching process [11]. The seed laser pulsewidth was 47 ps (FWHM), a value consistent with gain-switched operation of common diode lasers. Modelocked Ti-sapphire laser pulsewidths ranged from 21 to 39 ps (FWHM); pulsewidth increased with higher pump laser power.

Phase noise measurements were performed to quantify pulse timing jitter of the gain-switched diode laser master oscillator and modelocked Tisapphire slave laser. These measurements were made using an HP 8563B spectrum analyzer and vendor-supplied software. Integrated RMS phase noise $(10 \mathrm{~Hz}-1 \mathrm{MHz})$ was measured at the 15 th harmonic of the fundamental frequency. Measurements indicate that phase noise on the pulsed seed laser output is slightly less than $0.1^{\circ}$, a value

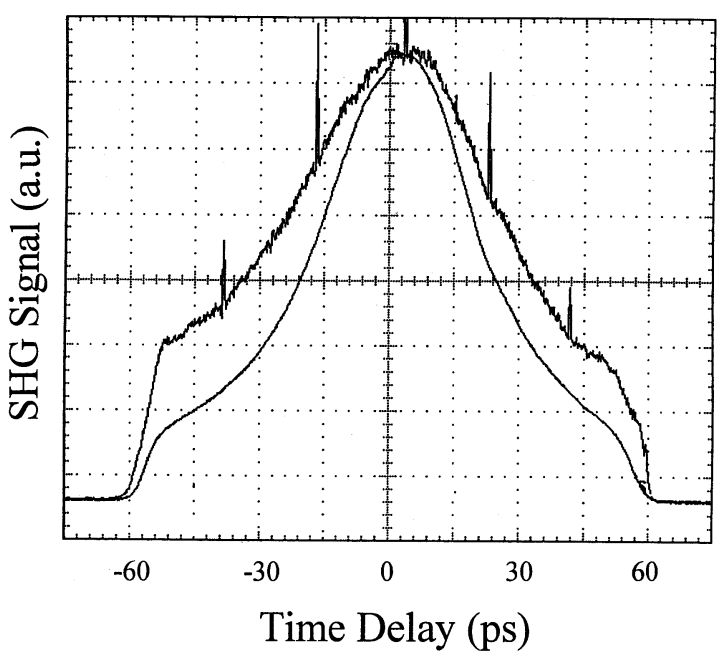

Fig. 3. Autocorrelator traces for the gain-switched diode seed laser (trace with spikes) and modelocked Ti-sapphire laser (smooth trace). To determine actual pulsewidth, the autocorrelation pulsewidth was multiplied by 0.707 to account for deconvolution of a Gaussian pulse shape. The clipped edges of the traces are not indicative of actual pulseshapes; the maximum temporal resolution of the autocorrelator is approximately 100 ps.

comparable to the phase noise of the synthesized source used to excite gain-switched operation. The integrated RMS phase noise of the modelocked Tisapphire laser output is $0.2^{\circ}$ which corresponds to timing jitter of $2.5 \mathrm{ps}$.

Although pulse-to-pulse amplitude fluctuations prevent the laser in its present state from being used for photoinjection at Jefferson Lab, it has a number of appealing features that warrant further research. The laser emits gigahertz pulse repetition rates and the average output power is higher than can be obtained from the diode laser system presently used $[2,12]$. The modelocked Ti-sapphire laser has low timing jitter as a result of injection seeding using a simple, stable gain-switched diode laser. We are presently designing a Ti-sapphire laser that incorporates active cavity length control in an effort to minimize amplitude noise on the Ti-sapphire laser output that may result from microphonic vibrations of various optical components. The new laser will also use an intracavity Faraday rotator to force unidirectional, traveling wave oscillation in 
an effort to reduce amplitude noise associated with spatial hole burning within the Ti-sapphire crystal. In addition, unidirectional traveling wave oscillation will provide for more efficient output coupling. With the present laser design, significant pulsed output is wasted through the input coupler $(\approx 40 \%)$. The new ring cavity, traveling wave Tisapphire laser will have one mirror that serves as both the output and input coupler. All of the pulsed output is usable because the input seed laser beam and Ti-sapphire laser output beam are not colinear when oscillation within the Ti-sapphire laser is unidirectional. In addition to the improvements mentioned above, the birefringent tuning filter will be reintroduced to the cavity to explore the wavelength tuning capabilities of the laser system. In conclusion, the results presented here provide "proof of principle" that injection modelocking using a gain-switched diode laser is a promising method to obtain high power, pulsed laser light with gigahertz pulse repetition rates.

\section{Acknowledgements}

This work was supported by the US Department of Energy Contract No. DE-AC05-84ER40150.

\section{References}

[1] K. Aulenbacher, in: K. Jager et al. (ed.), Proc. 12th Int. Symp. on High-Energy Spin Physics, World Scientific, Singapore, 1997.

[2] M. Poelker, Appl. Phys. Lett. 67 (1995) 2762.

[3] Various workshop proceedings detail existing and proposed electron accelerators. See for example the Proc. 1995 Particle Accelerator Conf. IEEE, New Jersey, 1995.

[4] D.B. Gustavson et al., Nucl. Instr. and Meth. 165 (1979) 177.

[5] B.E. Norum, T.P. Welch, CEBAF Letter of Intent, LOI93-012.

[6] L. Goldberg, D. Mehuys, D. Welch, IEEE Photon. Technol. Lett. 6 (1994) 1070.

[7] J. Hoffmann, P. Hartmann, C. Zimmermann, Nucl. Instr. and Meth. A 379 (1996) 15.

[8] S. Basu, P. May, J.M. Halbout, Opt. Lett. 14 (1989) 1272.

[9] See for example, P.T. Ho, in Picosecond Optoelectronic Devices, C.H. Lee (Ed.), Academic Press, New York, 1984.

[10] The wavelength of the modelocked Ti-sapphire laser was different from the diode seed laser by $5 \mathrm{~nm}$. This suggests that the Ti-sapphire laser is not a multipass amplifier as described in Ref. [8].

[11] E.P. Ippen, C.V. Shank, Ultrashort Light Pulses, in: S.L. Shapiro (Ed.), Springer, Berlin, 1977.

[12] M. Poelker, J. Hansknecht, in: R. Holt et al. (ed.), Conf. Proc. 7th Int. Workshop on Polarized Targets and Polarized Beams, AIP, New York, 1997.

[13] M. Poelker, Appl. Phys. Lett. 67 (1995) 2762. 